"This is the peer reviewed version of the following article: Ashcroft MB, Gollan JR, Ramp D (2014), Creating vegetation density profiles for a diverse range of ecological habitats using terrestrial laser scanning. Methods in Ecology and Evolution 5(3):263-272. doi:

10.1111/2041-210X.12157which has been published in final form at 10.1111/2041-

210X.12157. This article may be used for non-commercial purposes in accordance with Wiley Terms and Conditions for Self-Archiving." 
Received Date : 04-Jun-2013

Revised Date : 01-Dec-2013

Accepted Date : 16-Dec-2013

Article type : Research Article

\title{
Creating vegetation density profiles for a diverse range of ecological habitats using
} terrestrial laser scanning

\section{Running title: Vegetation density profiles using TLS}

Ashcroft, Michael B. ${ }^{1,2, *}$

Gollan, John R. ${ }^{1,3}$

Ramp, Daniel ${ }^{3}$

${ }^{1}$ Australian Museum, 6 College St, Sydney, NSW, Australia, 2010.

${ }^{2}$ Australian Wetlands, Rivers and Landscapes Centre, School of Biological, Earth and

Environmental Sciences, The University of New South Wales, NSW 2052, Australia

${ }^{3}$ University of Technology, Sydney, School of the Environment, PO Box 123 Broadway, New South Wales, 2007, Australia

* Corresponding author. Phone +61 (0)2 9320 6475. Fax +61 (0)2 93206012

\begin{abstract}
1. Vegetation structure is an important determinant of species habitats and diversity. It is often represented by simple metrics, such as canopy cover, height and leaf area index, which

This article has been accepted for publication and undergone full peer review but has not been through the copyediting, typesetting, pagination and proofreading process, which may lead to differences between this version and the Version of Record. Please cite this article as doi: 10.1111/2041-210X.12157
\end{abstract}

This article is protected by copyright. All rights reserved. 
do not fully capture three-dimensional variations in density. Terrestrial Laser Scanning (TLS) is a technology that can better capture vegetation structure, but methods developed to process scans have been biased towards forestry applications. The aim of this study was develop a methodology for processing TLS data to produce vegetation density profiles across a broader range of habitats.

2. We performed low-resolution and medium-resolution TLS scans using a Leica C5 Scanstation at four locations within eight sites near Wollongong, NSW, Australia (34.38$\left.34.41^{\circ} \mathrm{S}, 150.84-150.91^{\circ} \mathrm{E}\right)$. The raw point clouds were converted to density profiles using a method that corrected for uneven ground surfaces, varying point density due to beam divergence and occlusion, the non-vertical nature of most beams, and for beams that passed through gaps in the vegetation without generating a point. Density profiles were evaluated against visual estimates from three independent observers using coarse height classes (e.g. 5$10 \mathrm{~m})$.

3. TLS produced density profiles that captured the three-dimensional vegetation structure. Although sites were selected to differ in structure, each was relatively homogeneous, and yet we still found a high spatial variation in density profiles. There was also large variation between observers, with the RMS error of the three observers relative to the TLS varying from $16.2 \%$ to $32.1 \%$. Part of this error appeared to be due to misjudging the height of vegetation, which caused an overestimation in one height class and an underestimation in another.

4. Our method for generating density profiles using TLS can capture three-dimensional vegetation structure in a manner that is more detailed and less subjective than traditional methods. The method can be applied to a broad range of habitats—not just forests with open understoreys. However, it cannot accurately estimate near-surface vegetation density when there are uneven surfaces or dense vegetation prevents sufficient ground returns. Nonetheless,

This article is protected by copyright. All rights reserved. 
TLS density profiles will be an important input for research on species habitats, microclimates and nutrient cycles.

Key-words: canopy cover, habitat heterogeneity, leaf area index, LIDAR, TLS, vegetation structure

\section{Introduction}

Vegetation structure is an important characteristic of habitat for many species including mammals, amphibians, reptiles, birds and invertebrates (e.g. James \& Wamer 1982; Gunnarsson 1990; Werner \& Glennemeier 1999). The complexity or heterogeneity of vegetation can also affect collective properties of biodiversity, such as species diversity (Tews et al. 2004), which makes it a valuable indicator for monitoring programmes (Noss 1990). In addition, vegetation structure also has indirect effects on biodiversity by altering surface microclimates and nutrient cycles (Geiger 1971; Didham \& Lawton 1999; Ashcroft \& Gollan 2012).

Given these important and broad ranging impacts, it is unsurprising that ecologists have studied vegetation structure extensively, and many methods to quantify it have been developed and compared (Jennings et al. 1999; Fiala et al. 2006; Korhonen et al. 2006; Paletto \& Tosi 2009). However, vegetation structure is generally simplified to metrics such as leaf area index (LAI), canopy cover or vegetation height (e.g. Jennings et al. 1999; Wilson 2011). These metrics hide much of the three-dimensional complexity of vegetation structure (Lefsky et al. 2002), but are all that was practical using traditional methods such as visual estimates, hemispherical photographs, aerial photography, and optical/radar remote sensors (Jennings et al. 1999; Fiala et al. 2006; Korhonen et al. 2006; Paletto \& Tosi 2009). Recent advances in airborne (ALS) and terrestrial (TLS) laser scanning have now made it possible to 
capture the complete three-dimensional structure of vegetation (Lefsky et al. 2002).

Conventional metrics are still possible with laser scanning, but additional outputs can also include three-dimensional reconstructions of individual trees (Côté et al. 2009, 2012) or profiles that capture how vegetation density varies with height (Henning \& Radtke 2006; Zhao et al. 2013). These additional outputs from laser scanning contain more structural information than traditional methods and hence have great potential to improve the way vegetation structure is captured in ecological analyses. In this paper we describe a method for estimating vegetation density profiles using TLS so that we can capture more of the vegetation structure than is represented by traditional metrics.

Laser scanning, whether terrestrial or airborne, produces three-dimensional point clouds by emitting millions of laser beams and calculating the time taken for those beams to be reflected back by objects, such as the ground or vegetation (Lefsky et al. 2002; Dassot et al. 2011). Airborne sensors capture the top-down canopy architecture across broad landscapes from aeroplanes (e.g. Lefsky et al. 2002; Jenkins 2012), while terrestrial scanners are placed $\sim 1-2 \mathrm{~m}$ above the ground and capture the vegetation at an individual site, typically from below the tree canopy (e.g. Henning \& Radtke 2006; Danson et al. 2007; Seidel et al. 2012). Each method has advantages and disadvantages. For example, due to beam divergence and occlusion, ALS will typically produce a denser point cloud in the upper canopy but may not capture finer subcanopy elements or the ground surface, while TLS will capture a denser point cloud in the lower canopy but may not capture the complete upper canopy (Henning \& Radtke 2006; Van der Zande et al. 2006). In this paper we use TLS because density profiles will be affected by all subcanopy elements, including those that will be difficult to detect with ALS. However, this restricts our methodology to individual sites, whereas ALS could potentially be used over entire landscapes if this limitation could be overcome.

This article is protected by copyright. All rights reserved. 
A number of laser scanners are available including those that provide a single point from the first reflected surface, those that provide multiple returns where the laser is partially intercepted and reflected from multiple elements (e.g. Lovell et al. 2003; Durrieu et al. 2008), and those that provide a full-waveform of reflected energy over time (e.g. Jupp et al. 2009; Pirotti 2011; Zhao et al. 2013). Sensors that provide multiple returns or full-waveform potentially provide more information on canopy structure, but may be ambiguous as to whether the final return blocks the laser or whether the laser continues through a gap thereafter. Full-waveform lasers have been utilised to estimate density profiles from gap fractions, but these methods have typically focused on forestry applications and heights between the sensor and the top of the canopy (Jupp et al. 2009; Zhao et al. 2013). Their ability to capture a broader range of ecological habitats including shrubs and ground covers is yet to be assessed. In this paper we focus on the first-returns from lasers because these are commonly used (Lovell et al. 2003), but acknowledge that multiple-return or full-waveform instruments or techniques may also be used.

While TLS has great potential to produce density profiles, and thus improve how vegetation structure is captured, there are also a number of issues to overcome. First, the raw point clouds produced by laser scanners are biased by the divergence and occlusion issues mentioned above, and hence cannot be used directly in ecological analysis (Seidel et al. 2012). That is, the density of points in the raw TLS outputs are biased by proximity to scanner, and do not truly reflect vegetation density at any given height above the ground. A second issue is that the laser beams from terrestrial scanners are emitted in all directions, and are not all vertical beams like the virtual 'pins' that are used to define the cover or density of vegetation in any layer (Jennings et al. 1999; Wilson 2011). Angled beams will pass through a larger distance in any height layer than a vertical beam, and this could lead to an increasing 
overestimation of density at any height as the angle from vertical increases. A third issue is that some beams pass directly through the vegetation without being reflected, or are reflected at insufficient strength for the sensor to detect (Danson et al. 2007). Those beams that pass through the canopy do not produce a point, and hence do not affect TLS output, but must be considered when calculating densities or density will be overestimated. A fourth issue is that scanners must accurately capture the ground surface to determine how vegetation density changes with height, and although TLS may have advantages over ALS in this respect, it may still be difficult when dense vegetation and uneven ground allow few beams to reach the surface. A fifth issue is that wind may cause the vegetation to move, which may also cause scanners to overestimate the overall cover (Côté et al. 2009; Dassot et al. 2011). This is especially true if point clouds are converted to voxels (volumetric pixels, considered 'full' if they contain at least one scanned point) to correct for varying point density (e.g. Seidel et al. 2012), but is less problematic if densities are based on the proportion of beams that are reflected (Henning \& Radtke 2006).

Studies have now started addressing these above issues. For example, Henning \& Radtke (2006) calculated the percentage of beams reflected in each voxel to correct for varying point density, while Danson et al. (2007) used scans in closed rooms as a comparative means to estimate the number of beams that pass through canopies. However, there is still a bias towards forestry applications rather than broader ecological purposes (Vierling et al. 2008; Dassot et al. 2011), and there is still a need to develop methods that overcome all of the above issues and evaluate them across a variety of habitats. This is an important omission, because existing methods may only be effective in low-density or openunderstory stands (Dassot et al. 2011). For example, ALS has been shown to capture broad habitat types and accurately measure the upper canopy of trees (Lefsky et al. 2002; Bässler et

This article is protected by copyright. All rights reserved. 
al. 2011), but has difficulty quantifying the finer-scale structure of subcanopy trees and shrubs (Henning \& Radtke 2006; Dassot et al. 2011; Jenkins 2012). If we are only interested in forestry, then we may be able to develop and employ a method that only captures the structure of the large trees, but broader ecological applications also require data on other vegetative elements.

A secondary goal of this study was to examine the cost effectiveness of TLS for capturing density profiles. Traditional methods of estimating canopy cover have been assessed for time or cost effectiveness (e.g. Korhonen et al. 2006) but there are additional factors that need to be considered when using TLS. For example, increasing the resolution of the laser scan produces a higher density of points but increases scan time, while targets can be used so that multiple scans from different positions in the same site can be combined into one image to reduce issues of occlusion. We assessed whether the benefits of using higher resolution scans or targets justified the extra time commitment.

\section{Material and methods}

\section{TLS DATA COLLECTION}

We selected eight sites near Wollongong, NSW, Australia $\left(34.38-34.41^{\circ} \mathrm{S}, 150.84-150.91^{\circ} \mathrm{E}\right)$ to capture a broad range of vegetation structure. Sites included coastal dune shrublands and woodlands, temperate rainforests and eucalypt forests. The maximum height of vegetation at each site varied from $\sim 4 \mathrm{~m}$ to more than $30 \mathrm{~m}$, with canopy species including broad leaved species as well as those with needle-like leaves (e.g. Casuarina spp.). The cover of the upper strata varied from $\sim 30 \%-90 \%$. Understorey vegetation also varied in density and height, as illustrated in the colour photos of the profiles and canopies of sites that are included in Appendix S1. The eight sites were broadly classified as: 1 - open understorey tall forest; 2 - 
woodland with dense ferns; 3 - dense rainforest; 4 - open understorey woodland; 5 - shrubby woodland; 6 - swampy, needle-leaved woodland; 7 - low shrubland; and, 8 - mixed height shrubland.

The TLS scanner we used was a Leica C5 Scanstation. This scanner was used to perform two scans at four locations within each of the eight sites: a medium resolution scan $(\sim 14.7$ million points in $\sim 6$ minutes $)$ and a low resolution scan $(\sim 3.7$ million points in $\sim 2$ minutes). The scanner records only the point from the first return. The maximum range of the scanner was $35 \mathrm{~m}$, but to ensure the full profile was recorded even when distance from scanner was $\sim 10 \mathrm{~m}$, we restricted our analysis to heights of up to $30 \mathrm{~m}$. The scanner can recognise specific targets within an image, and these can be used to co-register multiple scans from each site into one combined image that reduces issues associated with occlusion. However, due to issues discussed later, co-registration was not eventually completed. We deployed a three inch square blue target with silver circle at the centre of each site, and four $\sim 20 \mathrm{~cm}$ diameter black and white circle targets were deployed in approximately a $5 \mathrm{~m}$ by $5 \mathrm{~m}$ square surrounding the central target (see Appendix S1). Some variation in target placement was needed to ensure targets were in open areas where they would be visible to the scanner, and the height of the scanner varied from $\sim 50 \mathrm{~cm}$ to $2 \mathrm{~m}$ to maximise visibility. Targets were scanned separately to the main scan, and in dense vegetation branches were held back during the target scans (but not the main scan) to maximise visibility. Targets were only scanned in the medium resolution scans.

\section{TLS METHODOLOGY}

Our goal was to produce density profiles for a range of habitats by addressing the five issues discussed in the introduction of this article (beam divergence and shading, angled beams, moving vegetation, pass-through beams and uneven ground surfaces). The first step was to

This article is protected by copyright. All rights reserved. 
define vertical pillars that we would calculate the density profiles for. We selected $5 \mathrm{~m}$ square pillars, with nine pillars arranged in a square with the laser scanner at the centre. Height of pillars was capped to $30 \mathrm{~m}$ due to the range of the scanner. Ground level was assumed to be equal to the lowest point in the TLS data within each of the pillars (as done by e.g., Henning \& Radtke 2006; Fig. 1). The pillar configuration was determined based on trial scans we obtained during a pilot study. Although smaller pillars would allow us to estimate ground level more accurately over uneven terrain, we found that a dense understorey could prevent beams reaching the ground if the pillars were small and this would cause us to overestimate the height of the ground surface. Similarly, we tried using pillars that extended further from the laser scanner, but found that a $3 \times 3$ arrangement of $5 \mathrm{~m}$ pillars was preferable, as pillars that were further from the scanner were also prone to inaccurate ground level or density estimates as the point density become lower. The nine $5 \mathrm{~m}$ pillars in each scan provided replication of density profiles, and also allowed us to quantify spatial variability in vegetation structure.

The second step in our analysis was to divide each pillar up into horizontal slices with a height of $25 \mathrm{~cm}$ (Fig. 1), where the $25 \mathrm{~cm}$ resolution was chosen to be larger than the positional accuracy of the scanner $(6 \mathrm{~mm})$ and maintain a balance between fine profile resolution and sufficient laser density. We aimed to determine the density of vegetation in each of these $5 \mathrm{~m} \times 5 \mathrm{~m} \times 25 \mathrm{~cm}$ prisms by calculating the proportion of beams that were reflected in each prism. To do this, we treated each point in the TLS scan as part of a laser beam, and divided each beam into segments of length $25 \mathrm{~cm}$ (equal to the height classes). That is, we assumed a beam passing through a distance of $25 \mathrm{~cm}$ at any angle had the same probability of being reflected as a vertical beam of $25 \mathrm{~cm}$. The segments further away from the scanner than the point in the image were effectively 'blocked' and provided no 
information on density, but still needed to be counted in the total number of segments in each prism. We incremented the 'reflected' count in the prism that contained the segment with the original point, and any segments prior to the reflected segment were added to the 'through' count for their respective prisms. Each of the counts (total, reflected, through) were calculated for each of the prisms.

The density has been estimated using the proportion of reflected pulses previously (e.g. Henning \& Radtke 2006), and this largely overcomes the problems associated with moving vegetation and beam divergence and occlusion. However, our variation of subdividing the beams into equal length segments provides an additional advantage. It overcomes the issue of beam angle, as angled beams are still assessed using the same distance as a vertical beam.

The third step was to apply a correction to cater for beams that passed through the vegetation without being reflected or creating a point. To do this, we repeated the above methodology using a scan taken in a closed room (as done by Danson et al. 2007). This was used to determine the total number of segments we expected in each prism. We then calculated the difference between the total segments from the closed room scan and the total segments from the vegetation scan, and added this to the 'through' total for the respective prism. The density of each prism could then be calculated as the number of reflected rays divided by the sum of reflected and through rays (Van der Zande et al. 2006; Danson et al. 2007). This estimate of density largely catered for the five issues mentioned previously (beam divergence and shading, angled beams, moving vegetation, pass-through beams and uneven ground surfaces), but there were still some limitations (see discussion). All analysis was done in R (R Development Core Team 2010; scripts included in Appendix S2).

This article is protected by copyright. All rights reserved. 
Once the density was known for each $25 \mathrm{~cm}$ high prism, the density could be calculated for broader height classes (e.g. 5-10 m) by multiplying the probabilities of a ray passing through each layer, where the probability was equal to one minus the density. We used the formula:

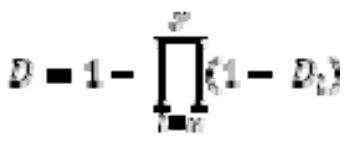

Where $D$ is the density of a combined layer that includes the $25 \mathrm{~cm}$ layers from height $x$ to height $y$, each with density $D_{l}$.

\section{COMPARISON WITH VISUAL OBSERVATIONS}

It is not possible to validate any estimate of vegetation structure accurately without destructive sampling (Danson et al. 2007). As destructive sampling was not feasible, and methods such as hemispherical photos do not provide any information on how vegetation density varies with height, we evaluated the TLS derived density profiles for our eight sites by comparing them against visual estimates. Three observers estimated the vegetation density at heights of 0-0.5 m, 0.5-1 m, 1-2 m, 2-5 m, 5-10 m and 10-30 m. To maximise consistency with the TLS methodology, density was defined as the number of imaginary vertical pins that would be intercepted by vegetation in each layer (Wilson 2011). Observers estimated the density at each of the eight sites without any discussion on results until after all estimates had been made. Visual estimates were based on a $20 \mathrm{~m}$ by $20 \mathrm{~m}$ square around the central target. TLS density profiles were compared against visual estimates to assess if they produced qualitatively similar results, and to calculate the RMS error for each observer.

This article is protected by copyright. All rights reserved. 


\section{Results}

The vegetation density profiles generally varied more between sites, than within sites (Fig. 2). Based on visual observations (see site photos in Appendix S1) these profiles appeared to be a good rendition of the density distribution at each site. The different profiles produced within each site also varied between the four scanner positions (Fig. 2) and between the nine columns analysed at each position (e.g. Fig. 3), demonstrating that even within our relatively homogeneous sites there was a large amount of spatial variation. However, there was little difference between the low and medium resolution scans at any given site and position (Fig. 2). That is, the density profile was affected by where we placed the scanner, but not the resolution of the scan we performed.

In general, there were no consistent differences between the density profiles of the nine pillars (as assessed using the average of all eight scans at all eight sites) that would suggest the density profiles were affected by the higher density of laser scans in the central pillar. However, in a number of cases where there was vegetation close to the scanner, the central pillar had much higher densities in the 1-3 m height range. This artefact was absent in most of the scans, suggesting the calculated density profiles were not biased by differences in laser density as long as there was no vegetation close to the scanner. Visual observations and the results from the four scanner positions (Fig. 2) support the fact that variations in density profiles capture spatial variation rather than biases between the nine pillars (Fig. 3).

Although we scanned targets as part of the medium resolution scans, they were not utilised in our analysis. This was mainly because scans needed to be processed individually to cater for the biases in point density caused by proximity to scanner (see methods), and it was

This article is protected by copyright. All rights reserved. 
not possible to perform such corrections if scans were combined such that beams were originating from multiple points in the combined image. However, it is possible to process images separately and then combine them together afterwards (unpublished data).

We still needed to assess the feasibility of targets to assess the cost effectiveness of different TLS scanning options (our secondary goal), and in particular the practicality of deploying targets in habitats with dense understoreys (a concern raised by Dassot et al. 2011). Scan time was affected by factors such as vegetation density, site access and operator experience, but to scan sites with four medium resolution scans and targets took $~ 90$ minutes per site, whereas four low resolution scans without targets took only $\sim 20$ minutes. The difference in scan time was minimal ( $\sim 6$ and 2 minutes per scan respectively), but targets needed to be scanned separately and individually from each of the four positions and this substantially added to the setup time.

Aggregating density profiles to coarse height ranges (i.e. $0-0.5 \mathrm{~m}, 0.5-1 \mathrm{~m}, 1-2 \mathrm{~m}, 2-$ $5 \mathrm{~m}, 5-10 \mathrm{~m}, 10-30 \mathrm{~m}$ ) reduced the amount of information on vegetation structure and made the differences between sites less obvious (compare Fig. 2 and Fig. 4). Nevertheless, this was necessary to compare the profiles against our three observers, as it was not feasible for visual observers to estimate density in fine height classes. The three observers predicted vegetation densities with average RMS errors of $17.5 \%, 31.2 \%$ and $21.6 \%$ respectively relative to the laser scanner (Fig. 5). The scanner was not necessarily predicting near surface densities accurately due to uneven surfaces and dense vegetation (see methods), but excluding the lower layer had little effect on these errors $(16.2 \%, 32.1 \%$ and $21.9 \%$ respectively).

This article is protected by copyright. All rights reserved. 


\section{Discussion}

\section{THE BENEFITS AND LIMITATIONS OF TLS}

In this study we developed a method to produce vegetation density profiles for different ecological habitats that varied in vegetation structure. Although we quantitatively assessed the method against visual observations, it should be stressed that there were apparent shortcomings of both methods and neither should be considered a true representation of vegetation structure. Therefore, this discussion examines the benefits and limitations of each method with reference to our results.

The primary benefit of TLS is that it can produce complex three-dimensional density profiles of vegetation structure. It was possible to examine finer resolution height classes (e.g. Fig. 2) and more spatial variation (e.g. Fig. 3) than was feasible with visual estimates (Fig. 5), and there was less subjectivity. While previous studies have highlighted that betweenobserver variability is a major shortcoming of visual estimates of canopy cover (Gorrod \& Keith 2009; Gollan et al. 2012), our results suggest this may be at least in part due to the inherent spatial variability of vegetation that can be quantified with TLS.

Another benefit of TLS is that it is a more accurate method of predicting height than our visual estimation technique. Indeed, some of the errors between TLS and observers appeared to be a direct result of observers underestimating the height of vegetation, as there was often an overestimation of vegetation in the 5-10 m height class and an underestimation in the 10-30 m height class (Fig. 5). This source of error in visual estimates could potentially be reduced if telescopic poles were utilised to determine more accurately the heights at each site. Other studies have also noted that lidar scanning can increase the accuracy of vegetation height estimates (Pirotti 2011), but heights can sometimes be underestimated by TLS if the density of points in the upper canopy is low (Durrieu et al. 2008).

This article is protected by copyright. All rights reserved. 
In developing our method, we also noted several shortcomings of TLS. First, the scanner and the targets had to be placed in relatively open positions within a given habitat, and this introduced a bias in where we were assessing canopy cover. Visual estimates were not as affected by this as we could move around to look at vegetation from different positions, and even deduce the density of vegetation in places that were not visible-a subjective approach that is not possible with TLS. It was apparent that TLS was usually underestimating density relative to observers (Fig. 5), and this may be partially because the laser scanner was biased towards open areas. We also noticed that that TLS overestimated density in the 1-3 m height range in the central column if there was vegetation close to the scanner.

The second shortcoming with TLS, at least in our analysis, is that it was not able to distinguish reliably between the ground surface and low vegetation. While full waveform or multiple return scanners are more likely to get a return from the surface, it is still difficult to determine if returns at slightly higher heights than this are from an uneven ground surface, loose rocks, woody debris, low vegetation, or small positional errors. Due to this issue, our estimates of the densities in the lowest layer, and possibly more on steeply sloped land, could be erroneous. This is not an issue for forestry applications if only large trees are of interest, but might be important for ecological applications if the density of shrubs and low ground covers are important. One potential solution to this is to use visual estimates for the lowest layers of vegetation, but rely on TLS for majority of the vegetation profile. It has been suggested that the strength of the reflected laser beam may be used to distinguish between leaves, woody structures or the ground (e.g. Côté et al. 2009). However, that was not tested in this study and it is not clear how accurate such a method would be using only one wavelength of laser beam (as opposed to a method such as hyperspectral remote sensing, Lillesand \& 
Kiefer 2000). Full-waveform sensors may also have greater ability to distinguish different target types (e.g. Jupp et al. 2009), but further research is needed to determine how well they can separate low vegetation from other objects.

A third shortcoming concerns the closed-room correction we applied to cater for beams that passed through the canopy without generating a return (a similar approach to that used by Danson et al. 2007). Unfortunately, there are also situations where beams do encounter an object, but are not reflected with sufficient strength to be detected by the scanner (Danson et al. 2007). This is problematic because we assume these beams passed through the canopy when they did not. This will result in an underestimation of vegetation density, and may be another reason why TLS gave lower densities than visual observers in our study.

A fourth limitation of TLS is applicable to analyses or methods that rely on targets to co-register multiple images for the same site. There have already been doubts raised about the feasibility of using targets in forested environments (Henning \& Radtke 2006), and our opinion is that it is not feasible across a broad range of habitats without introducing an unacceptable bias towards open understoreys to guarantee targets will be visible from multiple scanner positions. We identified there was a $\sim 75 \%$ time saving (20 minutes versus 90 minutes) when doing four low resolution scans without targets instead of four medium resolution scans with targets, and this saving would improve the feasibility of using TLS at many different sites. If we need to produce accurate images of trees (e.g. Côté et al. 2009, 2012) then targets may be necessary, but if we are only interested in producing density profiles then we can treat different scanner positions and columns as replicate estimates without having necessarily to co-register them into one combined image. Laser densities are

This article is protected by copyright. All rights reserved. 
configurable (not limited to low, medium and high) and future studies could investigate the trade-off between point densities and scan time in more detail. However, the scan time was not the major difference between our two scanning strategies, as target placement and scanning was the major time consuming task.

Not all of these limitations will be apparent for all potential applications. For example, in open understorey habitats the need to place the sensor in open areas will not necessarily introduce a bias. Similar, if the user is only interested in the structure of trees for forestry applications, then the errors in ground vegetation may not be a serious issue. However, many of these issues need to be addressed to make methods more appropriate for broader ecological research and applications.

\section{APPLICATIONS OF DENSITY PROFILES}

Density profiles can be interpreted to produce traditional metrics such as vegetation height and cover, but also contain extra information on how vegetation density changes in threedimensional space. This opens up new opportunities in how vegetation is incorporated into ecological research and management. For example, incoming solar radiation is intercepted by tree canopies, but the amount reaching the surface is affected by both the height and total cover (Breshears \& Ludwig 2010). Incorporating density profiles into these analyses could improve our ability to model sub-canopy radiation levels and consider the combined effect of different layers. Similarly, near-surface temperatures and humidities are also affected by canopy cover, but the height of the vegetation also needs to be considered (Ashcroft \& Gollan 2012). Using density profiles to determine the total net effect of the whole canopy structure on surface microclimates and energy balances is an important future application.

This article is protected by copyright. All rights reserved. 
Another potential application of density profiles is for vegetation monitoring or aboveground carbon assessments. Examination of the whole profile might detect changes that are not apparent in simple metrics such as height and LAI. Density profiles could also be utilised in studies of how vegetation structure directly affects the habitat suitability for different species (James \& Wamer 1982; Gunnarsson 1990). For example, different species may be sensitive to the structure or cover at a certain preferred height in the canopy (Shanahan et al. 2011), and density profiles allow researchers to focus on the cover at different heights for different species. Density profiles could also be used to assess bushfire hazard, for example, by detecting 'ladder fuels' that allow ground fires to reach the upper canopy (Lefsky et al. 2002). Density profiles provide more information on canopy structure than provided by traditional metrics, and these are just some of the potential applications that are yet to be fully realised by ecologists.

\section{Acknowledgements}

This project was funded by the New South Wales Government through its Environmental Trust (Grant\# 2011/RD/0099), with support from Australian Research Council Linkage Project LP100200080. University of Technology Sydney provided financial support to help purchase the Leica C5 Scanstation. Thanks to Emma McLeod for fieldwork assistance, Kresinda Turner for processing scan data, and to Andrew Letten and Evan Webster for help in the field during initial trial scans. Evan Webster also provided helpful discussion during the initial stages of the project.

This article is protected by copyright. All rights reserved. 


\section{References}

Ashcroft, M.B. \& Gollan, J.R. (2012) Fine-resolution (25 m) topoclimatic grids of nearsurface $(5 \mathrm{~cm})$ extreme temperatures and humidities across various habitats in a large $(200 \times 300 \mathrm{~km})$ and diverse region. International Journal of Climatology, 32, 21342148.

Bässler, C., Stadler, J., Müller, J., Förster, B., Göttlein, A. \& Brandl, R. (2011) LiDAR as a rapid tool to predict forest habitat types in Natura 2000 networks. Biodiversity and Conservation, 20, 465-481.

Breshears, D.D. \& Ludwig, J.A. (2010) Near-ground solar radiation along the grasslandforest continuum: tall-tree canopy architecture imposes only muted trends and heterogeneity. Austral Ecology, 35, 31-40.

Côté, J., Widlowski, J., Fournier, R.A. \& Verstraete, M.M. (2009) The structural and radiative consistency of three-dimensional tree reconstructions from terrestrial lidar. Remote Sensing of Environment, 113, 1067-1081.

Côté, J., Fournier, R.A., Frazer, G.W. \& Niemann, K.O. (2012) A fine-scale architectural model of trees to enhance LiDAR-derived measurements of forest canopy structure. Agricultural and Forest Meteorology, 166-167, 72-85.

Danson, F.M., Hetherington, D., Morsdorf, F., Koetz, B. \& Allgöver, B. (2007) Forest canopy gap fraction from terrestrial laser scanning. IEEE Geoscience and Remote Sensing Letters, 4, 157-160.

Dassot, M., Constant, T. \& Fournier, M. (2011) The use of terrestrial LiDAR technology in forest science: application fields, benefits and challenges. Annals of Forest Science, 68, 959-974.

This article is protected by copyright. All rights reserved. 
Didham, R.K. \& Lawton, J.H. (1999) Edge structure determines the magnitude of changes in microclimate and vegetation structure in tropical forest fragments. Biotropica, 31, 1730.

Durrieu, S., Allouis, T., Fournier, R., Véga, C. \& Albrech, L. (2008) Spatial quantification of vegetation density from terrestrial laser scanner data for characterization of 3D forest structure at plot level. Proceedings of SilviLaser 2008 (p. 325-334). Edinburgh, September 17-19, 2008, UK.

Fiala, A.C.S., Garman, S.L. \& Gray, A.N. (2006) Comparison of five canopy cover estimation techniques in the western Oregon Cascades. Forest Ecology and Management, 232, 188-197.

Geiger, R. (1971) The Climate Near the Ground. Revised edition. Harvard University Press, Cambridge, MA, USA.

Gollan, J., de Bruyn, L.L., Reid, N. \& Wilkie, L. (2012) Can volunteers collect data that are comparable to professional scientists? A study of variables used in monitoring the outcomes of ecosystem rehabilitation. Environmental Management, 50, 969-978.

Gorrod, E.J. \& Keith, D.A. (2009) Observer variation in field assessments of vegetation condition: implications for biodiversity conservation. Ecological Management and Restoration, 10, 31-40.

Gunnarsson, B. (1990) Vegetation structure and the abundance and size distribution of spruce-living spiders. Journal of Animal Ecology, 59, 743-752.

Henning, J.G. \& Radtke, P.J. (2006) Ground-based laser imaging for assessing threedimensional forest canopy structure. Photogrammetric Engineering \& Remote Sensing, 72, 1349-1358.

James, F.C. \& Wamer, N.O. (1982) Relationships between temperate forest bird communities and vegetation structure. Ecology, 63, 159-171.

This article is protected by copyright. All rights reserved. 
Jenkins, R.B. (2012) Airborne laser scanning for vegetation structure quantification in a south east Australian scrubby forest-woodland. Austral Ecology, 37, 44-55.

Jennings, S.B., Brown, N.D. \& Sheil, D. (1999) Assessing forest canopies and understorey illumination: canopy closure, canopy cover and other measures. Forestry, 1, 59-73.

Jupp, D.L.B, Culvenor, D.S., Lovell, J.L., Newnham, G.J., Strahler, A.H. \& Woodcock, C.E. (2009) Estimating forest LAI profiles and structural parameters using a ground-based laser called 'Echidna®'. Tree Physiology, 29, 171-181.

Korhonen, L., Korhonen, K.T., Rautiainen, M. \& Stenberg, P. (2006) Estimation of forest canopy cover: a comparison of field measurement techniques. Silva Fennica, 40, 577588.

Lefsky, M.A., Cohen, W.B., Parker, G.G. \& Harding, D.J. (2002) Lidar remote sensing for ecosystem studies. Bioscience, 52, 19-30.

Lillesand, T.M. \& Kiefer, R.W. (2000) Remote Sensing and Image Interpretation. Fourth edition. John Wiley \& Sons, NY, USA.

Lovell, J.L., Jupp, D.L.B., Culvenor, D.S. \& Coops, N.C. (2003) Using airborne and groundbased ranging lidar to measure canopy structure in Australian forests. Canadian Journal of Remote Sensing, 29, 607-622.

Noss, R.F. (1990) Indicators for monitoring biodiversity: a hierarchical approach. Conservation Biology, 4, 355-364.

Paletto, A. \& Tosi, V. (2009) Forest canopy cover and canopy closure: comparison of assessment techniques. European Journal of Forest Research, 128, 265-272.

Pirotti, F. (2011) Analysis of full-waveform LiDAR data for forestry applications: a review of investigations and methods. iForest, 4, 100-106.

R Development Core Team (2010) R: A Language and Environment for Statistical Computing. R Foundation for Statistical Computing.

This article is protected by copyright. All rights reserved. 
Seidel, D., Fleck, S. \& Leuschner, C. (2012) Analyzing forest canopies with ground-based laser scanning: a comparison with hemispherical photography. Agricultural and Forest Meteorology, 154-155, 1-8.

Shanahan, D.F., Possingham, H.P. \& Martin, T.G. (2011) Foraging height and landscape context predict the relative abundance of bird species in urban vegetation patches. Austral Ecology, 36, 944-953.

Tews, J., Brose, U., Grimm, V., Tielbörger, K., Wichmann, M.C., Schwager, M. \& Jeltsch, F. (2004) Animal species diversity driven by habitat heterogeneity/diversity: the importance of keystone structures. Journal of Biogeography, 31, 79-92.

Van der Zande, D., Hoet, W., Jonckheere, I., van Aardt, J. \& Coppin, P. (2006) Influence of measurement set-up of ground-based LiDAR for derivation of tree structure. Agricultural and Forest Meteorology, 141, 147-160.

Vierling, K.T., Vierling, L.A., Gould, W.A., Martinuzzi, S. \& Clawges, R.M. (2008) Lidar: shedding new light on habitat characterization and modeling. Frontiers in Ecology and the Environment, 6, 90-98.

Werner, E.E. \& Glennemeier, K.S. (1999) Influence of forest canopy cover on the breeding pond distributions of several amphibian species. Copeia, 1, 1-12.

Wilson, J.B. (2011) Cover plus: ways of measuring plant canopies and the terms used for them. Journal of Vegetation Science, 22, 197-206.

Zhao, F., Yang, X., Strahler, A.H., Schaaf, C.L., Yao, T., Wang, Z., Román, M.O., Woodcock, C.E., Ni-Meister, W., Jupp, D.L.B., Lovell, J.L., Culvenor, D.S., Newnham, G.J., Tang, H. \& Dubayah, R.O. (2013) A comparison of foliage profiles in the Sierra National Forest obtained with a full-waveform under-canopy EVI lidar system with the foliage profiles obtained with an airborne full-waveform LVIS lidar system. Remote Sensing of the Environment, 136, 330-341.

This article is protected by copyright. All rights reserved. 


\section{Author contributions}

MA, JG and DR were involved in developing concepts for the project. MA \& JG conducted the fieldwork. MA developed methods for processing the TLS data, wrote the R scripts, did the analysis and led the writing. JG \& DR contributed to the final manuscript.

\section{Supporting Information}

Additional Supporting Information may be found in the online version of this article.

Appendix S1. Colour photographs of the eight sites scanned in this study.

Appendix S2. R code for producing density profiles.

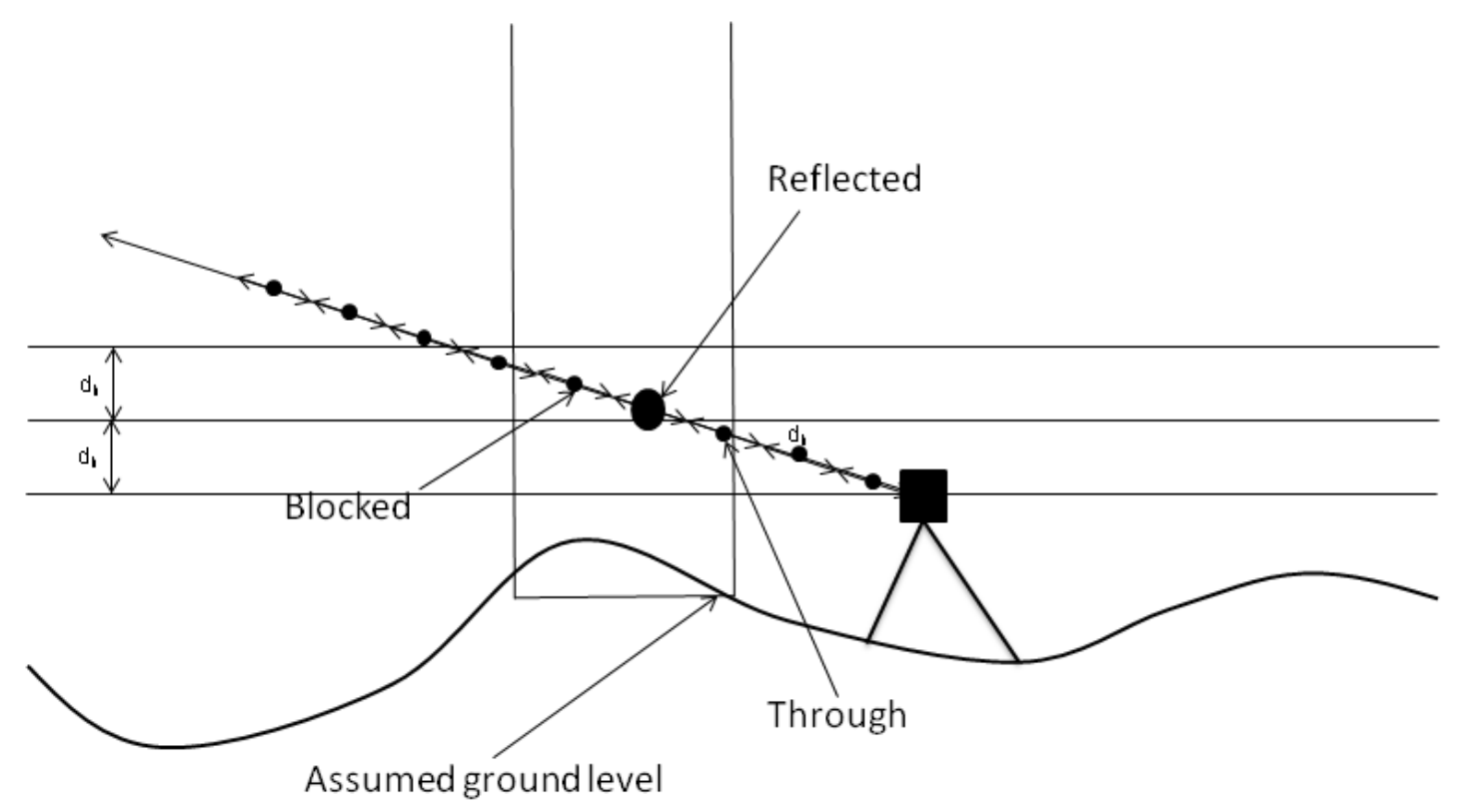

Fig. 1. The method for produced density profiles consisted of dividing the scan into $5 \mathrm{~m}$ by 5 m pillars (e.g. vertical lines in figure), estimating ground level using the lowest return in each pillar, and then dividing each pillar into prisms of $25 \mathrm{~cm}$ height (e.g. horizontal lines in figure). Each point in the TLS data was converted to a ray, and broken into segments of equal 
length to the height classes $(25 \mathrm{~cm})$. The density in each prism was then calculated by determining the proportion of segments that were reflected (see methods for full details).
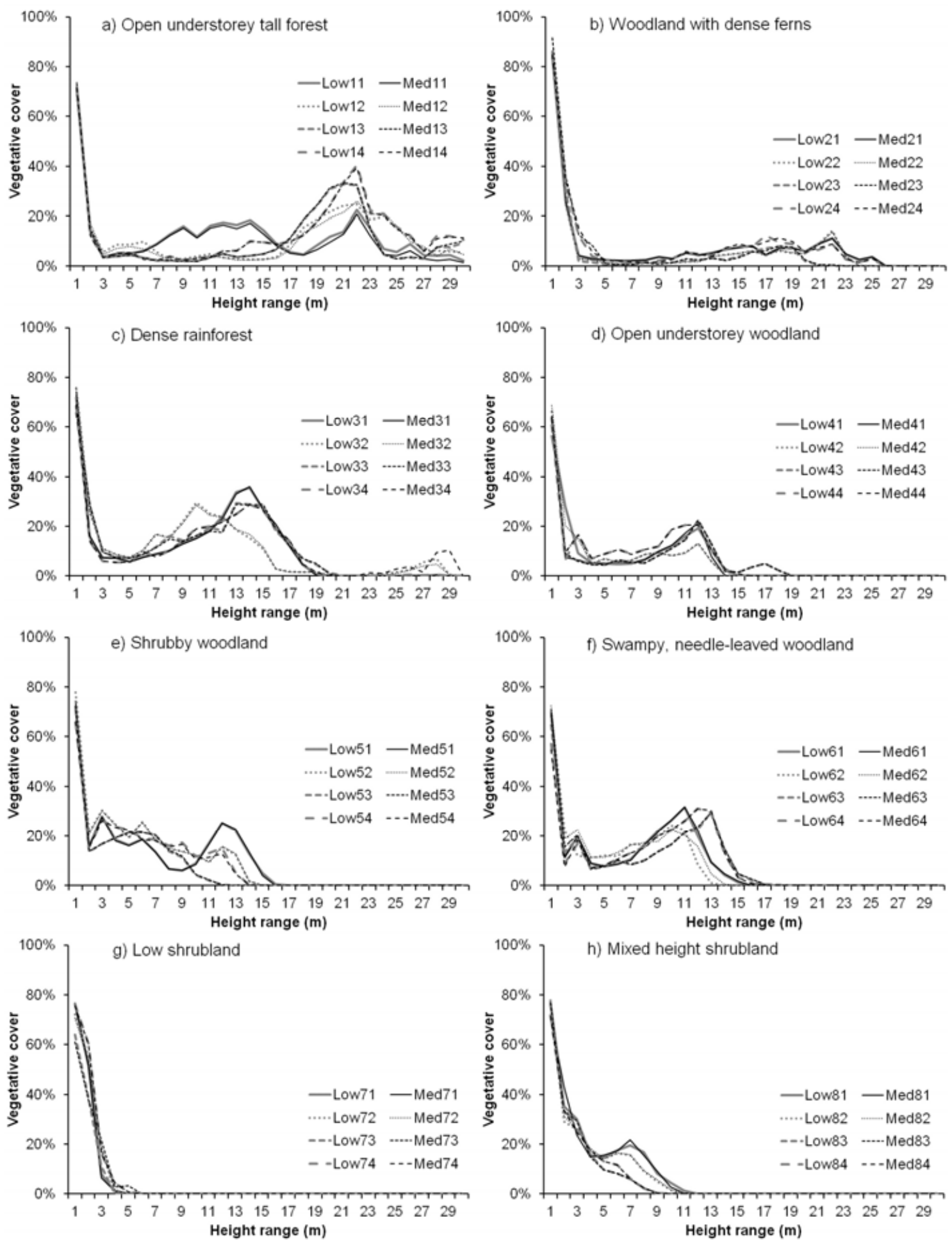

This article is protected by copyright. All rights reserved. 
Fig. 2. The density profiles produced from low-resolution ('Low') and medium ('Med') resolution scans at four positions (last number in legends) within eight sites (first number in legends). The raw output in $25 \mathrm{~cm}$ height classes has been aggregated to $1 \mathrm{~m}$ height classes.

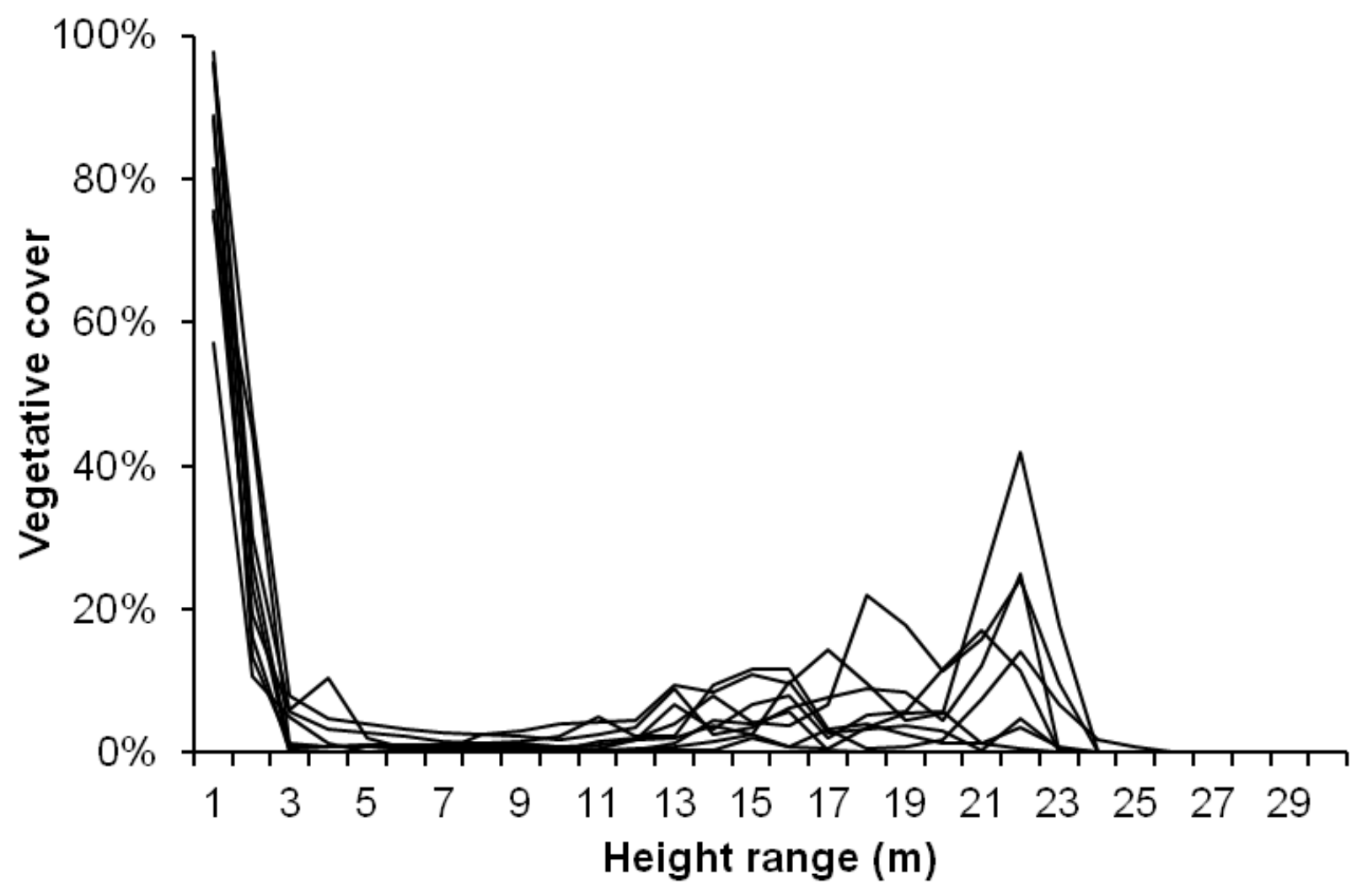

Fig. 3. The density profiles produced from the nine different pillars form the medium resolution scan at the second position of the second site. These are the components of the average density profile 'Med22' in Fig. 1. The raw output in $25 \mathrm{~cm}$ height classes has been aggregated to $1 \mathrm{~m}$ height classes.

This article is protected by copyright. All rights reserved. 

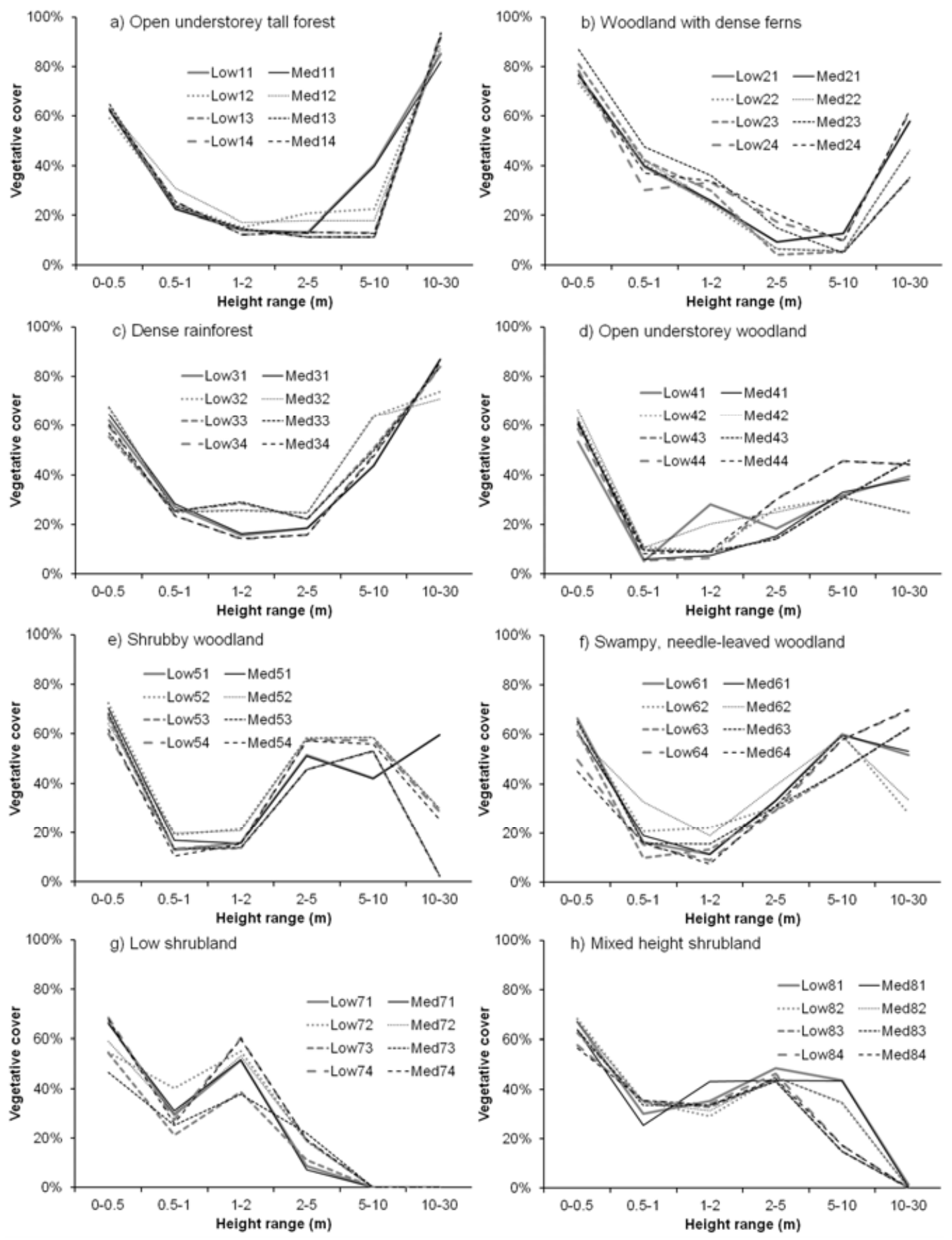

Fig. 4. The density profiles produced from low-resolution ('Low') and medium ('Med')

resolution scans at four positions (last number in legends) within eight sites (first number in

legends). The raw output in $25 \mathrm{~cm}$ height classes has been aggregated to broader height

classes for comparison with visual observers.

This article is protected by copyright. All rights reserved. 

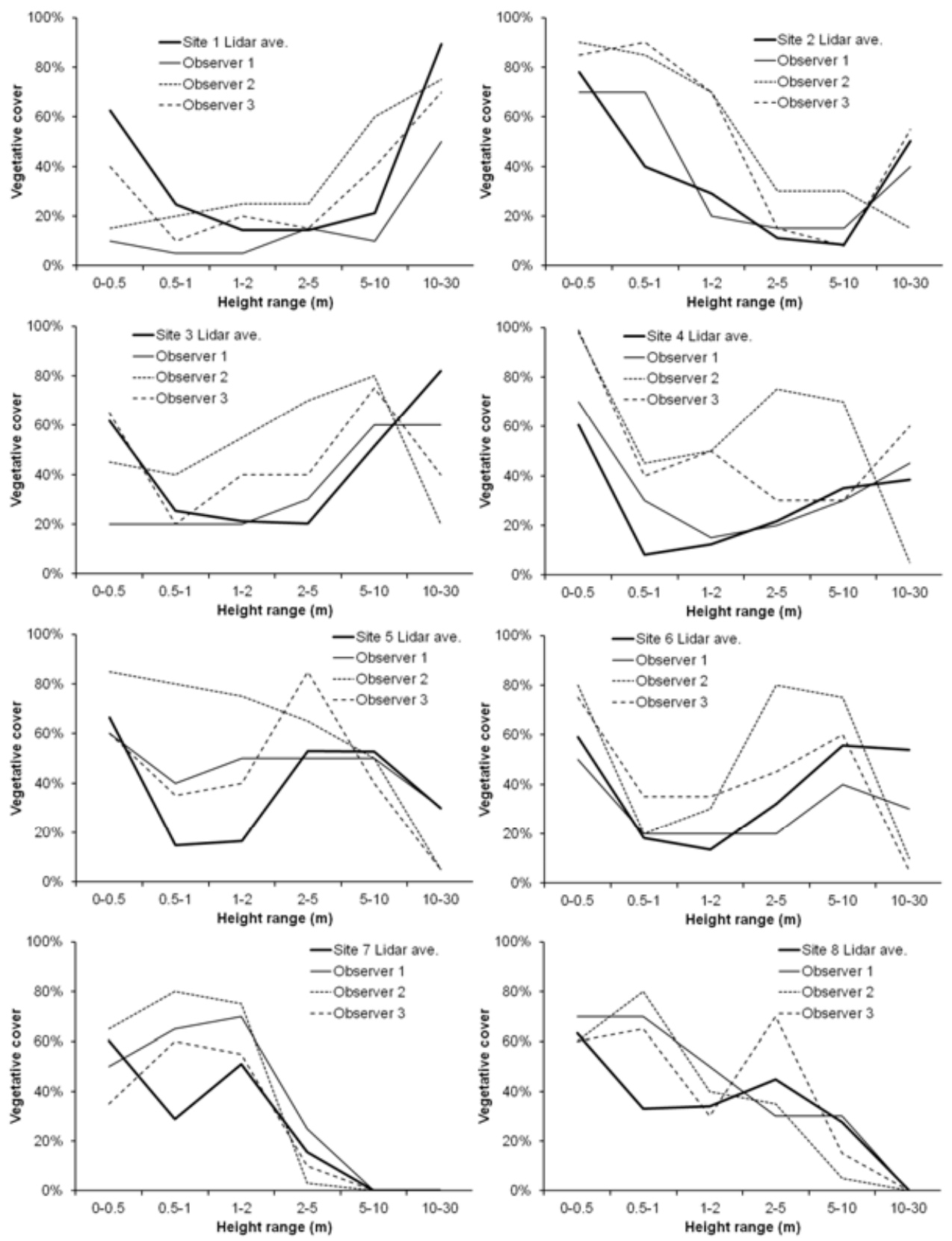

Fig. 5. The density profiles estimated by three observers in comparison to the average density profile produced by TLS at eight sites. The raw TLS output in $25 \mathrm{~cm}$ height classes has been aggregated to broader height classes for comparison with visual observers.

This article is protected by copyright. All rights reserved. 